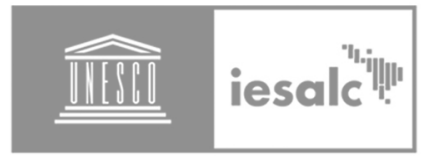

\title{
Unesco
}

Instituto Internacional

para la Educación Superior

en América Latina

y el Caribe
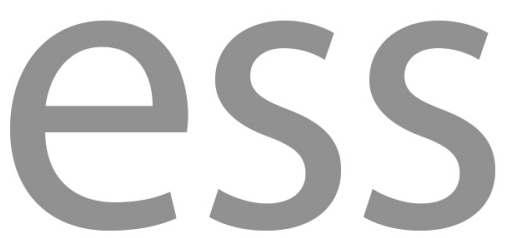

?

Educación

Superior y

Sociedad

Vol. 33 No. 2 (2021)

-

$\bullet$

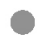

$\bullet$
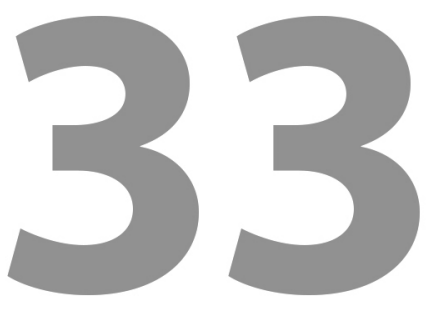

Incluye Dossier Temático:

Desafíos de la educación superior frente a la pandemia de Covid-19 en América Latina y el Caribe 


\section{Educación remota de emergencia: experiencias de estudiantes de una unidad académica regional de la BUAP durante la pandemia de COVID-19}

Abelardo Romero Fernández, Laura Villanueva Méndez,

Fabio Morandín Ahuerma, Ana Liviere Vargas Vizuet

\section{RESUMEN}

Este estudio presenta los resultados de una investigación cuantitativa de alcance exploratorio que tuvo por objetivo recuperar y explorar las experiencias de los estudiantes de nivel licenciatura, que tuvieron que concluir el semestre de primavera 2020 por medio de clases no presenciales con los medios que disponían durante la etapa de mayor incertidumbre sobre la pandemia generada por Covid-19. Se aplicó un cuestionario con preguntas cerradas y abiertas a 124 estudiantes de las licenciaturas de Estomatología, Medicina General y Comunitaria, Nutrición Clínica y de Psicología de una unidad académica regional en la zona nororiental del Estado de Puebla. Entre los resultados se destaca que, aunque la mayoría de los estudiantes cuenta con un servicio de internet para realizar sus tareas y acceder a sus clases, se señala como problema constante para cubrir objetivos de aprendizaje la baja calidad en conexión a internet. Además, se identifica que aunque en la mayoría de los casos se revisaron los temas de los programas de estudio, los estudiantes consideraron que no se cubrieron los objetivos de aprendizaje, lo que relacionaron principalmente a la aplicación de técnicas y actividades no adecuadas para las clases no presenciales por parte de los docentes. Finalmente la falta de práctica clínica fue un elemento que presentó diferencia significativa en la licenciatura en estomatología respecto a las otras licenciaturas en el logro de objetivos de aprendizaje.

Palabras Clave: Educación Superior; Covid-19; Regionalización; Educación Remota de Emergencia; Experiencias de Estudiantes. 


\title{
Emergency Remote Teaching: experiences of students from a BUAP Regional Academic Unit during the Covid-19 Pandemic
}

\begin{abstract}
This study presents the results of a quantitative research with exploratory scope that aimed to recover and explore the experiences of undergraduate students who concluded semester in spring 2020 through virtual classes with the means available during the stage of greater uncertainty related to Covid-19 pandemic. A questionnaire with closed and open questions was applied to 124 students from a regional academic unit in the northeastern area of the State of Puebla studying the following degrees: Stomatology, General and Community Medicine, Clinical Nutrition and Psychology. Among the results, it is highlighted that, although most of the students have an internet service to carry out their homework and access their classes, the low quality of internet connection is pointed out as a constant problem to meet learning objectives. In addition, it is identified that although in most cases the topics of the study programs were reviewed, the students considered that the learning objectives were not covered, which was mainly related to the application -by teachers- of techniques and activities not suitable for virtual classes. Finally, the lack of clinical practices was an element that presented a significant difference in the degree in Stomatology in the achievement of learning objectives, compared to other degrees.
\end{abstract}

Key words: Higher Education; Covid-19; Regionalization; Remote Emergency Education; Student Experiences.

\section{Educação remota emergencial: experiências de estudantes na Unidade Acadêmica Regional BUAP durante a Pandemia de Covid-19}

\section{RESUMO}

Este estudo apresenta os resultados de uma pesquisa quantitativa de abordagem exploratória que objetivou resgatar e explorar as experiências de alunos de graduação que concluiram o semestre na primavera de 2020 em aulas virtuais com os meios disponíveis durante o período de maior incerteza em relação com a pandemia gerada pela Covid-19. Questionário com perguntas fe- 
chadas e abertas foi aplicado a 124 alunos dos cursos de Estomatologia, Medicina Geral e Comunitária, Nutrição Clínica e Psicologia de uma unidade acadêmica regional do nordeste do Estado de Puebla. Dentre os resultados, destaca-se que, embora a maioria dos alunos possuía serviço de internet para realização dos deveres de casa e acesso às aulas, a baixa qualidade da conexão à internet é apontada como um problema constante para o cumprimento dos objetivos de aprendizagem. Além disso, embora na maioria dos casos os tópicos dos programas de estudos foram revistos, os alunos consideraram que os objetivos de aprendizagem não foram contemplados, o que esse fato estava relacionado principalmente à aplicação pelos docentes de técnicas e atividades não adequadas para aulas não presenciais. Finalmente a falta de prática clínica foi um elemento que apresentou uma diferença significativa em Estomatologia no cumprimento dos objetivos de aprendizagem, em relação aos demais cursos.

Palavras-chave: Ensino Superior; Covid19; Regionalização; Educação Remota Emergencial; Experiências do Aluno.

\section{Enseignement à distance em situation d'urgence: expériences d'étudiants d'une unité académique régionale du BUAP pendant I a pandémie de Covid-19}

\section{RESUMÉ}

Cette étude présente les résultats d'une recherche quantitative à portée exploratoire qui visait à récupérer et à explorer les expériences d'étudiants de premier cycle qui ont conclu le semestre au printemps 2020 à travers des cours non présentiels avec les moyens dont ils disposaient pendant la période de plus grande incertitude en relation à la pandémie générée par le Covid-19. Un questionnaire avec des questions fermées et ouvertes a été appliqué à 124 étudiants des diplômes de licence en Stomatologie, Médecine Générale et Communautaire, Nutrition Clinique et Psychologie d'une unité universitaire régionale du nordest de l'État de Puebla. Parmi les résultats, il a été signalé que, bien que la majorité des étudiants disposent d'un service internet pour faire leurs devoirs et accéder à leurs cours, la faible qualité de la connexion internet est montrée comme un problème constant dans la poursuite des objectifs d'apprentissage. En outre, il a été identifié que, bien que dans la plupart des cas les sujets des programmes 
d'études aient été revus, les étudiants ont considéré que les objectifs d'apprentissage n'étaient pas couverts, ce qui était principalement lié à l'application de techniques et d'activités non adaptées aux classes virtuelles mises en place par les enseignants. Finalement, le manque de pratique clinique a été un élément qui a démontré une différence significative en Stomatologie dans l'atteinte des objectifs d'apprentissage, par rapport aux autres diplômes.

Mots clés: Enseignement Supérieur; Covid-19; Régionalisation; Enseignement à distance em situation d'urgence; Expériences Étudiantes.

\section{INTRODUCCIÓN}

El lunes 16 de marzo del 2020 alrededor de las 12:00 horas comenzó a circular por medio de redes sociales, como Facebook y WhatsApp, un mensaje de las autoridades de la Benemérita Universidad Autónoma de Puebla (BUAP) sobre la suspensión de clases en toda la universidad debido a la contingencia sanitaria causada por COVID-19. Nadie comprendía muy bien lo que implicaba esta suspensión o cuanto duraría. Al comienzo se creía que dicha suspensión de actividades presenciales duraría unas semanas y que se regresaría a clases después de las vacaciones de Semana Santa (13 de abril). Sin embargo, poco a poco se fue extendiendo el periodo de suspensión y ante la incertidumbre, la BUAP creó un Comisión Institucional para el Seguimiento y Evaluación para la Pandemia por el SARS CoV-2, COVID-19, que evaluaba medidas que se debían poner en práctica para contener los contagios en la universidad.

En este escenario se comenzó a considerar, que lo más apropiado sería continuar con las actividades académicas del semestre de primavera 2020 de manera no presencial, esperando que para el mes de mayo se pudiera regresar a las actividades presenciales y concluir con el trabajo académico y los procesos de evaluación del semestre. No obstante, esto no fue posible y tanto los estudiantes como los docentes tuvieron que adaptarse a una nueva dinámica de trabajo improvisando medios y recursos digitales para proseguir con los cursos que se habían quedado a la mitad. Es importante mencionar que la suspensión de las actividades presenciales por COVID - 19 en la BUAP, se dio justo el día antes al regreso de las suspensiones de clases del viernes 13 y lunes 16 de marzo, como se 
marca en el calendario oficial de la universidad. Con esta suspensión de 2 días se creó una suspensión larga, que hizo que muchos estudiantes regresaran a sus hogares para pasar tiempo con sus familias, muchos de ellos dejaron sus materiales de estudio, equipos de cómputo, etc. sin saber que no regresarían en mucho tiempo.

Este artículo presenta los resultados de la primera etapa de un estudio que busca comparar cuales son las condiciones y problemáticas que los estudiantes tuvieron al inicio de la pandemia con las clases no presenciales y compararlos con los que tienen un año después de la suspensión de clases presenciales. Es importante mencionar que posteriormente al periodo de primavera 2020, la BUAP tomó acciones como capacitar a sus docentes en el uso de plataformas digitales, homologó el uso de software educativo, facilitó equipos de cómputo y recursos económicos a estudiantes y docentes, implementó convocatorias para el rediseño de los programas de asignaturas presenciales a modalidades no presenciales; entre otras acciones. Se espera acceder a aplicar un instrumento a la misma población, para conocer cómo se han adaptado los estudiantes a sus clases no presenciales y tener evidencia de si las medidas tomadas por la universidad han sido pertinentes y sobre todo si han logrado dar acceso a los estudiantes a una educación superior pertinente al contexto generado por la pandemia.

\section{Educación remota de emergencia en educación superior}

Esta no es la primera vez que las Instituciones de Educación Superior (IES) han tenido que cerrar sus puertas y continuar con sus funciones a pesar de enfrentar una contingencia sanitaria. De acuerdo con el Instituto Internacional para a Educación Superior en América Latina y el Caribe (IESALC) (2020), en 1666 la Universidad de Cambridge tuvo que suspender sus actividades presenciales debido a la epidemia de peste negra que sacudió Inglaterra, pero no detuvo sus actividades académicas. Es evidente que el cierre de los espacios físicos de las IES tendrá efectos a corto y largo plazo. De acuerdo con Toquero (2021) en el corto plazo, tanto docentes como estudiantes tuvieron que mover toda su dinámica presencial a un modelo educativo no presencial haciendo uso de cualquier medio que les permitiera seguir con sus actividades académicas; esto implicó un cambio y adaptación a una nueva cultura y medios ambientes de aprendizaje. 
Por otro lado, de acuerdo con el Banco Mundial (World Bank, 2020), las suspensiones prolongadas de las actividades presenciales en las IES representan un detrimento de aprendizaje que puede acrecentar la pérdida de capital humano y, finalmente, disminuir las oportunidades económicas en el largo plazo. IESALC (2020), estimaba que el cierre temporal afectaría aproximadamente a 23,4 millones estudiantes de educación superior y a 1,4 millones de profesores en Latinoamérica y el Caribe; lo cual es aproximadamente más del $98 \%$ de la población de estudiantes y profesores de educación superior de la región. De acuerdo con la Asociación Nacional de Universidades e Instituciones de Educación Superior (ANUIES) (2020), el total de la población matriculada en algún programa de educación superior en México es de 4,9 millones de estudiantes (el $4.7 \%$ del total de los estudiantes en Latinoamérica y el Caribe). La presente situación sin duda tendrá efecto negativo en la creación de capital humano cualificado a largo plazo que permita el desarrollo de la economía del país en una sociedad del conocimiento que ya no sólo depende del trabajo, la tierra y el capital como medio para generar desarrollo y bienestar para su población.

La emergencia sanitaria creada por el COVID-19, puso en jaque a la gran mayoría de las IES en México debido a que ninguna de ellas esperaba la suspensión de clases presenciales de forma tan repentina e indefinida. Esto causó que todas las instalaciones y espacios físicos pertenecientes a las universidades quedaran deshabilitados para su uso de un día para otro y que tanto la docencia, la investigación y la vinculación con la sociedad tuvieran que llevarse a cabo desde casa y por medio de instrumentos digitales improvisados.

El campus ha sido desplazado mayormente por estrechos espacios habitacionales donde los universitarios atienden, a través de una pantalla, las actividades propuestas por sus docentes. Asimismo, las tareas de investigación y extensión se han visto modificadas de una manera radical por el cierre de las instalaciones físicas y por el supuesto general de que la vida académica puede transcurrir entre pantallas, chats y correos electrónicos (Casanova Cardel, 2020, p. 13).

Tanto el personal docente como administrativo y estudiantes se tuvieron que adaptar a una modalidad que más que educación en línea podría ser llamada 
Educación Remota de Emergencia (ERE). Hodges et al. (2020) consideran que la educación en línea es el resultado de un cuidadoso proceso de diseño, planificación, sistematización y desarrollo. Además, cada elemento y decisión que se incluye o excluye tiene un impacto en la calidad de la educación. Estos autores también destacan que un cambio abrupto de modalidad presencial a modalidad en línea, como el causado por COVID-19, causará que la educación no cuente con procesos diseñados y planeados; lo cual tendrán un efecto en la calidad de la educación proporcionada. Además, Hodges et al. (ob.cit.) destacan que en la educación en línea se cuenta con papeles y dinámicas de interacción e integración bien definidas entre los docentes, los estudiantes, los objetivos, los materiales y los procesos de evaluación de las asignaturas que buscan crear ambientes apropiados para incrementar el aprendizaje. Usualmente, para que un curso de modalidad en línea sea puesto en marcha se debe planear y diseñar por un periodo de seis a nueve meses. Esto quiere decir que la educación en línea es mucho más que sólo contar con internet y un equipo de cómputo por medio del cual conectarse a una sesión en Zoom y recibir instrucciones de un docente. Por otro lado, la ERE se diferencia de la educación en línea, porque básicamente es el resultado de un cambio abrupto de un sistema educativo presencial a uno que debe improvisar medios y recursos para el cumplimiento de las necesidades educativas de los estudiantes; es decir no cuenta con una estructura definida y ésta se va creando de acuerdo a los recursos con los que se cuenta en el momento. Para Bozkurt et al. (2020) la ERE se trata de la sobrevivencia de un sistema educativo presencial por medio de todos los recursos disponibles ya sean en línea o no. De acuerdo con el Instituto Nacional de Estadística, Geografía e Informática (INEGI) (INEGI, 2019) en México, 80,626,159 personas son usuarios de internet, esto es el $70.1 \%$ de la población total del país. Del $100 \%$ de las personas con acceso a internet, el $89 \%(72,032,107)$ se conectan a internet desde sus hogares mientras que el $10.7 \%(8,594,052)$ lo hacen desde fuera de su hogar; ya sea por medio de datos móviles o lugares donde pueden rentar un equipo de cómputo. Sin embargo, a pesar de que en México $70.1 \%$ de la población tiene acceso a internet su uso era básicamente para acceder a información $(90.7 \%$ del $100 \%$ del uso al acceso a internet), para comunicarse $(90.6 \%$ del $100 \%$ de uso al acceso a internet), como medio de entretenimiento ( $91.5 \%$ del $100 \%$ de uso al acceso a internet), como apoyo a la educación o capacitación (83.8\% del 100\% de uso al acceso a internet) y para acceder a redes sociales ( 97.8 del $100 \%$ de 
uso al acceso a internet). De acuerdo a los datos presentados, el internet se usaba básicamente para actividades que tenían poco que ver con un aprendizaje formal, en todo caso se usaba como un apoyo para la educación o capacitación, pero no como el medio donde las personas recibieran educación formal.

\section{La educación de remota de emergencia en una unidad académica regional de la BUAP durante la pandemia de Covid-19}

Las IES han recurrido a la regionalización, como un medio para descentralizar y al mismo tiempo aumentar la oferta y cobertura de la educación superior en los llamados "desiertos educativos" para impulsar la creación de capital humano, la transferencia de conocimiento, la innovación tecnológica para el desarrollo local (Rivera Polo y Rivera Vargas, 2018). Castellar (2020) destaca que para que un país tenga mejores oportunidades de desarrollo, sus IES deben enfocarse en la calidad de la educación que ofrecen a su sociedad y que ésta a su vez se verá reflejada en crecimiento y desarrollo de la capacidad para producir conocimiento e innovación, lo que se manifiesta en el desarrollo de perfiles profesionales y de educación que aumenta la productividad de una región o un país. Sin embargo, Mejía-Pérez y Worthman (2018) han señalado que la calidad de unidades académicas regionales tiende a la baja debido a factores como la falta de recursos económicos y el bajo capital cultural de los estudiantes que ingresan a estas unidades académicas (que propician la deserción de los estudiantes), la dificultad para la contratación docentes (y ni hablar de la apertura de plazas de tiempo completo, que permitirían contar con una planta docente estable y con la posibilidad de acceder a programas de estímulos docentes por medio de una mejora constante de sus funciones educativas, investigativas y de vinculación con la comunidad) y por último, la falta de recursos y condiciones adecuadas en las instalaciones de las unidades académicas regionales. Todas las IES en el mundo se han tenido que adaptar a la nueva realidad creada por COVID-19, sin embargo, es importante tener un acercamiento a cómo vivieron este proceso los estudiantes desde las unidades académicas regionales, donde ya las condiciones son difíciles para lograr egresar de la educación superior.

De acuerdo con Ordorika (2020), los estudiantes de nivel licenciatura tuvieron que reorganizar y ajustar su vida personal con su vida académica. En la mayoría de los casos, tuvieron que regresar a casa y hacer frente a la nueva modalidad 
educativa de emergencia que la contingencia sanitaria por COVID-19 con los recursos que tuvieran a su alcance. América Latina cuenta con el $45 \%$ de conectividad desde el hogar y sólo supera a África que tiene el $17 \%$, es muy probable que muchos estudiantes de nivel superior no tengan acceso a internet si pertenecen a una zona rural de México. Además, tener una conexión a internet no es sinónimo de contar con una conexión de buena calidad que permita acceder a plataformas para participar de sesiones sincrónicas con el docente y compañeros o contenidos en línea; es muy común que en América Latina exista una brecha digital entre las zonas urbanas y las rurales que contribuye a incrementar la brecha académica IESALC (2020).

Por otro lado, los estudiantes tuvieron que adaptarse a nuevas dinámicas improvisadas por sus docentes por medio de nuevas plataformas, apps o software para "salvar" el semestre porque a pesar de que los estudiantes pudieran haber aprobado las asignaturas del semestre de primavera 2020, es muy posible que no desarrollaran completamente las competencias profesionales que requieren de la práctica en laboratorios, clínicas, empresas o instituciones. El solo hecho de que los docentes se vieran en la necesidad de continuar con sus clases por medio digitales improvisados y que los estudiantes también tuvieran que aprender a usar distintas plataformas o software para continuar con sus clases, indica que existía una curva de aprendizaje muy pronunciada respecto al uso de tecnología en educación superior. Al parecer la forma más fácil de continuar y concluir los cursos durante el semestre de primavera 2020 fue por medio de la transmisión de sesiones por video, sin que esto implique un ambiente de aprendizaje real; sino sólo la transmisión de conocimiento e instrucciones a los estudiantes.

Otro aspecto importante de la continuación de las clases no presenciales por medio de la ERE, es la que las IES buscaban concluir los cursos del semestre y esto implicaba que los estudiantes debían ser evaluados. Marinoni et al. (2020), reportaron que en mayo de 2020 la mayoría de las IES a nivel mundial tenían como prioridad asegurar que sus estudiantes aprobaran el semestre para obtener los créditos planeados, la prioridad era que los estudiantes no perdieran el semestre para no interferir en sus rutas académicas y el $89 \%$ de las IES, que participaron en el estudio, contemplaban a los exámenes como medio para la evaluación del semestre. Es importante prestar atención a como se concluyó el semestre de primavera 2020 respecto a las evaluaciones que se realizaron, debido a que esto pudo ser una causa de deserción por parte de los estudiantes 
de las mismas. En México la población total matriculada en educación superior en el periodo 2019-2020 era de 4.061 .644 y en ese entonces se tenía $7.4 \%$ de deserción, es decir 300,561 estudiantes abandonaban sus estudios de educación superior (SEP, 2020). Sin embargo, aunque la deserción se puede dar durante toda la trayectoria académica de los estudiantes, Silva (2020) distingue que la población estudiantil en mayor peligro de abandonar sus estudios se encuentra en el primer año de la licenciatura; esto puede haberse incrementado con las clases no presenciales.

Definitivamente las condiciones creadas por la pandemia de COVID-19 han llevado a la educación a tener características más cercanas a la ERE que a un modelo de educación en línea formal. Este estudio tiene por objetivo reportar cual fue la experiencia de estudiantes de una unidad académica regional de la BUAP localizada en la sierra nororiental de Puebla, al cambiar de sistema de educación superior presencial a lo que es conocido como ERE. La pandemia de COVID-19 ha llevado tanto a docentes como a estudiantes a salir de la zona de confort, desarrollar nuevas habilidades para no sólo cumplir con planes y programas educativos, sino para realmente desarrollar nuevos escenarios donde el aprendizaje no sólo sea enciclopédico y acumulativo, sino realmente significativo y valioso para la sociedad.

\section{METODOLOGÍA}

El presente trabajo presenta la primera parte del proyecto para explorar el impacto de la ERE durante la contingencia COVID-19 en una unidad académica regional de la BUAP ubicada en la Sierra Nororiental del Estado de Puebla que se divide en dos partes. Los resultados presentados, corresponden a la primera parte del proyecto con un estudio cuantitativo transversal de alcance exploratorio, que indagó las experiencias de los estudiantes de las licenciaturas de Estomatología, Medicina General y Comunitaria, Nutrición Clínica y Psicología al concluir el periodo Primavera 2020. Para recabar la información, se diseñó un cuestionario en línea de 27 preguntas cerradas y abiertas, organizadas en las siguientes seis dimensiones: conectividad a internet, experiencias de ERE durante la pandemia COVID-19, problemas de la ERE, percepción de logro de aprendizajes, diferencias entre clases presenciales y no presenciales y aporte 
de la tecnología a la educación no presencial. El cuestionario fue elaborado a partir de una revisión a la bibliográfica disponible respecto al impacto del COVID-19 en la educación superior.

El cuestionario fue respondido de forma anónima y voluntaria por 124 estudiantes después de los primeros tres meses de clases no presenciales del periodo Primavera 2020 a través de formularios de Google. Los 124 estudiantes representan el $22.1 \%$ de un total de 559 estudiantes que estaban inscritos en segundo, tercero, cuarto, quinto o sexto semestre de las licenciaturas de Estomatología, Medicina General y Comunitaria (MGC), Nutrición Clínica y Psicología. No se incluyó a estudiantes de semestres avanzados, debido a que las materias que ellos cursaban se relacionan a otras actividades como servicio social, prácticas profesionales y prácticas clínicas que no se llevan a cabo en la institución o que se encontraban suspendidas.

\section{RESULTADOS}

\section{Perfil general de los estudiantes y conectividad}

El $74 \%$ de los participantes de este estudio fueron mujeres y el $26 \%$ fueron hombres, estudiantes de las licenciaturas de Estomatología, Medicina General y Comunitaria, Nutrición Clínica y Psicología que en su mayoría cursaba el segundo, cuarto o sexto semestre de su carrera (92.7\%). Un gran porcentaje (45\%) de ellos son originarios de la zona Nororiental del Estado de Puebla o del Estado de Veracruz (47\%).

El 83\% de los estudiantes manifestaron tener acceso a internet. De los estudiantes con acceso a internet, poco más de la mitad (55\%) acceden desde su servicio de internet en casa, un $20 \%$ accede por medio de telefonía celular con datos móviles y un $18 \%$ tiene que acudir a un sitio público con costo. El porcentaje restante de estudiantes, accede a internet desde casa de familiares, vecinos o en el lugar de trabajo (7\%). Los dos dispositivos más utilizados por los estudiantes para acceder a internet son el teléfono celular (51\%) y computadora portátil (41\%).

Experiencias durante la educación remota de emergencia durante la pandemia COVID-19

Sobre el porcentaje de temas cubiertos durante el semestre Primavera 2020, el $29.8 \%$ consideró que se cubrió entre el 71 y $80 \%$ de contenidos, el $25 \%$ consideró que se cubrió el $60 \%$ o menos de los contenidos, mientras que el $22.6 \%$ 
consideró que se cubrió entre el 61 y $70 \%$ de los contenidos. Aunque el porcentaje de contenidos cubiertos supera el $50 \%$, en la mayoría de los casos, los estudiantes (79\%) declararon que consideran que no obtuvieron aprendizajes significativos durante el periodo Primavera 2020.

La percepción de la falta de aprendizaje se puede explicar a partir de diferentes factores, entre los que destacan: el acceso a internet o a Tecnologías de la Información y Comunicación (TIC) (calidad y estabilidad de acceso a internet y software), didácticas y técnicas no apropiadas para el trabajo en línea y la falta de interacción docente-estudiante durante las clases no presenciales.

\section{Principales problemas durante la educación remota de emergencia}

Los estudiantes de todas las licenciaturas reportaron que uno de los principales problemas durante el periodo de Primavera 2020 en clases no presenciales fue el acceso a internet con un $27 \%$, que contrasta con la accesibilidad del $83 \%$ de estudiantes que declararon tener acceso a internet. Lo anterior tiene una relación con la región de la cual son originarios, ya que uno de los problemas comunes en la región Nororiental del Estado de Puebla y zonas cercanas, caracterizadas como regiones rurales o semiurbanas, es la baja calidad de la conexión a internet, lo cual concuerda con la brecha de acceso a internet señalada por el INEGI (2019) en México de 28.9 puntos porcentuales de diferencia de acceso a internet en poblaciones urbanas y rurales. El estudio de Ramos Mancilla (2019), sobre el acceso a internet en la Región Norte de Puebla resalta aspectos de baja velocidad de internet en municipios como Ixtepec y Xochitlan, que pertenecen a la Sierra Nororiental, que propician el bajo acceso al mismo. Además, señala a los cortes constantes de electricidad debidos a tormentas como un problema que influye en el acceso a internet. La nota periodístíca sobre acceso a internet en la Sierra Norte de Puebla (González, 2020) menciona entre las principales problemáticas de acceso a clases virtuales, la mala señal de internet proporcionada por las compañías de comunicación de telefonía celular de la región y cabe señalar que la Sierra Nororiental del Estado de Puebla comparte características geográficas y sociales con la Sierra Norte. De esta manera es común, que aunque los estudiantes cuenten con servicios de internet en casa o datos móviles de navegación, los cortes constantes o la baja velocidad repercutan en la evaluación de la calidad del aprendizaje percibido por ellos como lo mencionan algunos estudiantes: "... hubo contratiempos en las entregas de trabajo, y algunos profesores 
no entendían que por una u otra razón la red no era tan buena. En mi caso, el problema es que vivo en una comunidad y hay veces en que se va la luz" (estudiante de Psicología, segundo semestre), "...el internet , aunque tuviera, luego cuando llueve se va la luz" (estudiante de MGC, cuarto semestre), “...se iba la señal de internet de mi casa muy seguido y tuve que acudir a vecinos, o al ciber para poder tomar clase, hacer exámenes y subir trabajos" (estudiante de Estomatología, segundo semestre),"que se trababa mi internet y a veces los profesores comenzaban y cuando yo entraba ya no sabía ni de que estaba hablando" (estudiante de Nutrición Clínica, cuarto semestre).

El 11.3\% de los estudiantes mencionó como principal problemática, la adaptación al uso de diferentes plataformas y software utilizado por los docentes. Lo anterior coincide con la falta de la homologación de plataformas por parte de la institución durante el periodo Primavera 2020, cuando los docentes tuvieron que improvisar medios y actividades para continuar con el semestre, de ahí que los estudiantes reporten una variedad de plataformas utilizadas al mismo tiempo para las diferentes asignaturas como medio principal para llevar a cabo las clases no presenciales: Classroom (100\%), Facebook (79\%), WhatsApp (76.6\%), Microsoft Teams (62\%), Edmodo (54.8\%) y Google Meets (37.1\%) principalmente. Mientras que plataformas para fomentar ambientes de aprendizaje virtual como Moodle (0.8\%) o Blackboard (18.5\%) fueron mencionados por una minoría de estudiantes. Esto muestra como redes sociales y plataformas gratuitas fueron los medios que se utilizaron más durante la emergencia sanitaria.

\section{Percepción de logro de aprendizajes y razones asociadas}

Como se mencionó antes, el 79\% de los estudiantes consideró no haber obtenido aprendizajes significativos. Entre las razones que comparten para dar esta respuesta, destaca la aplicación de técnicas y actividades no adecuadas para las clases no presenciales por parte de los docentes (22.6\%), ya que estuvieron centradas en la revisión autónoma de temas por parte de los estudiantes como: entrega de trabajos, lecturas y reportes, lo que se relaciona a que un $25 \%$ de las menciones hacen referencia a la saturación de tareas como una característica de las clases no presenciales. Al respecto, algunos mencionaron "en realidad no tomamos clases, solo nos mantuvimos con actividades para ir abarcando los temas" (estudiante de Estomatología, segundo semestre), " ...la saturación de tareas y que en varias ocasiones las asignaciones eran para la misma fecha" (es- 
tudiante de Nutrición Clínica, cuarto semestre), "la cantidad de tareas que dejaban, llegó un punto donde eran demasiadas que me causó mucho estrés, frustración" estudiante de Psicología, cuarto semestre), "los doctores se enfocaban mas en dejar tareas que en llevar la clase como tal" (estudiante de MGC, cuarto semestre), en algunos casos, las tareas son percibidas por los estudiantes como evidencias no significativas para su aprendizaje:

porque la mayoría de los maestros, solo dejaban tareas y tareas y todas las querían en pocos días, entonces se me acumulaban y por hacerlas y terminar a tiempo no entendía del todo lo que estaba haciendo y algunos maestros ya ni sabían que pedir porque ya habíamos terminado el programa y por eso solo pedían tareas de relleno o repetidas (estudiante de Estomatología, cuarto semestre).

A la saturación de tareas se sumaron, las responsabilidades y actividades del entorno familiar y no tener condiciones adecuadas en casa para estudiar (14.8\%), como lo señalan algunos: "que debía cuidar a mi hermano y prima, por ello es que me estresaba más" (estudiante de Psicología, segundo semestre), "ahora tengo que colaborar en las labores domésticas y se me hace muy complicado" (estudiante de Nutrición Clínica, segundo semestre). Mencionaron también la falta de comprensión de los temas y actividades solicitadas debido al uso de estas plataformas o aplicaciones "...era algo muy nuevo para mi, aprender a usar cada una de las plataformas y al hacer las tareas asignadas tener dudas y no poder resolverlas" (estudiante de MGC, cuarto semestre), "tomar ritmo a las plataformas, que había algunas que no sabíamos cómo se utilizaban" (estudiante de MGC, sexto semestre).

En relación a las técnicas y actividades no adecuadas para el aprendizaje, los estudiantes también identifican a la falta de retroalimentación (23.4\%) de las actividades y temas por parte de sus docentes como un elemento central de la falta de aprendizajes significativos "la razón por la cual puse que no, fue porque los catedráticos únicamente se comunicaban con nosotros para mandar la carga de trabajo sin retroalimentar los temas o resolver dudas" (estudiante de Estomatología, cuarto semestre), "usualmente cada clase va acompañada de una explicación que parcial o totalmente resuelve nuestras dudas y en estos casos no había" (estudiante de Nutrición Clínica, sexto semestre), "existían varios maes- 
tros que solo se dedicaban a enviar tareas adjuntando PDFs los cuales no entendía y cuando se tenía alguna duda el maestro no respondía" (estudiante de Psicología, cuarto semestre).

\section{Diferencias entre clases presenciales y no presenciales}

Finalmente, sobre las diferencias entre clases presenciales y no presenciales para el logro de aprendizajes, señalaron a la falta de interacción docente-estudiante como un problema, lo que se relacionó al perfil profesional de los estudiantes encuestados, ya que ellos (33\%) consideraron que la licenciatura que estudian, demanda conocimientos que deben adquirirse con la práctica y observación directa de un experto, aspecto que se vio limitado durante este periodo. Además de considerar que en sus licenciaturas es necesario acudir a laboratorios o espacios especiales:"somos del área de la salud, necesitamos dominar temas de gran importancia, ver los temas completos, trabajamos con la salud de las personas" (estudiante de MGC, sexto semestre), "considero que en mi licenciatura es demasiado importante la práctica clínica, la cual debería ser presencial" (estudiante de Estomatología, sexto semestre), "En mi carrera se necesita hacer muchas practicas lo cual es casi imposible realizar por medios digitales" (estudiante de Estomatología, cuarto semestre). Para comparar diferencias entre las licenciaturas respecto a la importancia que le atribuyen a las clases presenciales en el logro de objetivos de aprendizaje se aplicó una prueba $\mathrm{X}^{2}$ en donde se encontró una diferencia significativa en la licenciatura en Estomatología $\left(X^{2}\right.$ con $\left.p=0.012\right)$. Dos estudiantes de Estomatología lo expresan:

cada carrera es diferente, en el caso de Estomatología es $50 \%$ práctico y $50 \%$ teórico, pero depende mucho del semestre en el que estés. Los primeros 2 (años) creo que se pueden tomar por línea sin mucho problema, pero los demás, es más difícil porque llevas laboratorio (el cual necesitas practicar bastante) y a partir de sexto entras a clínica, donde forzosamente necesitas interacción con paciente, aunque en estos tiempos de COVID, es muy riesgoso porque por más que lleves guantes, cubrebocas y todo lo necesario, hay probabilidades de que atiendan a alguien que ya esté contagiado (estudiante de sexto semestre). 
las prácticas en laboratorio, ya que los docentes no pueden corregirte tan fácilmente a través de la computadora, por ejemplo en los detalles anatómicos, como los dientes son pequeños, a veces no es posible distinguir bien la imagen, porque se pixelea (estudiante de sexto semestre).

Una diferencia importante entre clases presenciales y no presenciales que impactó en el logro de aprendizajes fue que los horarios de las clases eran irregulares o no respetados por los docentes durante las clases no presenciales (18.6\%): "tener que estar todo el día atento a los mensajes de whats, las apps de las sesiones en línea, etc. por si mandaban alguna indicación, estar todo el día frente a la computadora por las clases" (estudiante de Nutrición Clínica, segundo semestre), algunos estudiantes identificaron como causa de la saturación de tareas y falta de respeto a los horarios a que los docentes también tenían que enviar evidencias de trabajo:

el cambio de horario de algunos docentes que chocaban con otra materia y no respetaban el horario ya establecido, el que un docente de un día para otro nos pedía trabajo porque a ellos le pedían evidencia y en semanas no nos enviaba algún trabajo, más cuando le pedían evidencia a ellos (estudiante de Estomatología, sexto semestre).

\section{Aporte de la tecnología a la educación no presencial}

El 100\% de los estudiantes mencionó haber hecho uso de la tecnología para continuar con el semestre de forma no presencial, sin embargo, el $46.8 \%$ consideró que la tecnología agregó valor al posibilitar la realización de las actividades propuestas por los docentes pero no generó cambios significativos en la forma de trabajo y el aprendizaje debido a que fue utilizada sólo para enviar tareas y calificaciones (18.5\%): "la mayoría de las tareas y actividades encargadas se hicieron en la libreta y todo se escaneaba y se enviaba por medio de las plataformas" (estudiante de MGC, segundo semestre), "las tareas a veces no eran calificadas, y solo las hicieron para tener llena la plataforma" (estudiante de Psicología, cuarto semestre). Algunos señalaron que con el uso de tecnología, los docentes empeoraron la forma en la que impartín sus clases (11.3\%) como lo señala una estudiante de MGC: 
no utilizaron ninguna estrategia de aprendizaje como deberían con la tecnología de hoy en día, fue igual que en presenciales, lectura de diapositivas y explicación con falta de aire, tareas (más de lo normal), algunas veces cancelaba clases siendo en línea porque estarían ocupados, pdf que leer y comprender por nuestra cuenta y videos (en ocasiones), no hubo ninguna mejora, la diferencia fue exageración de tareas (que no calificaron todas porque ya era mucho trabajo y grupos para ellos y no podían con tanto), y si fallaba el internet no era culpa suya (quinto semestre).

El 16\% consideró que gracias al uso de tecnología no perdieron contacto con sus docentes y pudieron acceder a información por medio de bases de datos:

la tecnología si nos fue de mucha ayuda para poder contactar y tener comunicación pero no se supo aprovechar del todo porque solo pocos maestros si daban clases en línea y estaban al pendiente de nosotros y nos proporcionaban material para reforzar lo visto en clase, pero otros solo enviaban tareas así que no creo que hubiera un gran cambio en la enseñanza de algunos maestros (estudiante de Psicología, sexto semestre).

\section{DISCUSIÓN Y CONCLUSIONES}

A partir de la revisión de los resultados, se identificaron algunos problemas durante el periodo Primavera 2020, que correspondió a la primera etapa de suspensión de actividades académicas presenciales. Una situación reportada por los estudiantes, fue la baja conexión a internet a pesar de contar con un servicio de internet en casa o datos de telefonía celular.

Esta situación se relaciona a la brecha de acceso a internet entre comunidades rurales y urbanas de México señalada por IESALC (2020) y por el INEGI (2019). Lo anterior cobra especial interés debido a que la Región Nororiental del Estado de Puebla y zonas cercanas de las que proviene la mayoría de los estudiantes, se caracteriza como región rural o semirural, sin embargo cabe señalar que para el periodo Otoño 2020, la institución tomó entre otras medidas, el préstamo de 
equipo de cómputo y de paquetes de datos de internet a estudiantes que lo solicitaron.

Uno de los resultados más preocupantes, fue que el cumplimiento de la revisión de los temas de las asignaturas no se relacionó a una percepción de logro de aprendizaje por parte de los estudiantes y esto puede relacionarse a la importancia del papel docente en el proceso de enseñanza-aprendizaje, ya que entre las razones para la baja percepción de logro de aprendizaje se encuentran: la saturación de tareas asignadas por los docentes, la implementación de técnicas o actividades no adecuadas por parte de docentes, el uso de diferentes e inadecuadas plataformas para las clases no presenciales, la falta de revisión de tareas y retroalimentación de actividades. Estos resultados enfatizan la importancia de la capacitación y actualización docente tanto en el uso de plataformas educativas y en estrategias pedagógicas en actividades no presenciales. Congruente con esto, la institución implementó una serie de acciones encaminadas a mejorar las condiciones educativas en el periodo Otoño 2020 como: oferta de cursos y talleres enfocados al uso de las plataformas Blackboard, Moodle y Microsoft Teams para docentes y estudiantes, se contrataron plataformas educativas y se homologó su uso en las distintas unidades académicas, se emitió un documento de lineamientos para las clases no presenciales en que se enfatizó la importancia de respetar horarios, mantener contacto con los estudiantes, fomentar el trabajo autónomo sin descuidar el seguimiento de cada uno de ellos y mostrar flexibilidad frente a los casos que lo requirieran.

Finalmente destaca también la diferencia encontrada en la Licenciatura en Estomatología respecto a la importancia de la práctica clínica para el logro de los aprendizajes y las dificultades para suplir esta práctica con las actividades no presenciales. Será muy interesante, en la segunda parte de este estudio, identificar las estrategias y acciones que se hayan tomado en periodos subsecuentes de actividades no presenciales. 


\section{REFERENCIAS}

ANUIES (2020). Anuario estadístico de la población escolar en educación superior: ciclo escolar 2019-2020. http://www.anuies.mx/informacion-y-servicios/informacion-estadistica-de-educacion-superior/anuario-estadistico-de-educacion-superior

Castellar, E. (2020). Una mirada al estado de las Instituciones de educación superior con relación a los objetivos de desarrollo sostenible. Educación Superiory Sociedad, Vol. 32 (2), pp.14-35.

https://www.iesalc.unesco.org/ess/index.php/ess3/index

González, C. (23 abril de 2020). Alumnos de la Sierra Norte de Puebla sin internet ni computadora para hacer frente a las clases virtuales en la contingencia del Covid-19.

https://www.periodicocentral.mx/2020/gobierno/item/8454-alumnos-de-la-sierra-norte-de-puebla-sin-internet-ni-computadora-para-hacer-frente-a-las-clases-virtuales-en-la-contingencia-delcovid-19\#ixzz6qAyaFwNa

Bozkurt, A., Jung, I., Xiao, J., Vladimirschi, V., Schuwer, R., Egorov, G. \& Paskevicius, M. (2020). A global outlook to the interruption of education due to COVID-19 pandemic: navigating in a time of uncertainty and crisis. Asian Journal of Distance Education, 15(1), pp.1-126. https://doi.org/10.5281/zenod0.3878572

Hodges, C., Moore, S., Lockee, B., Trust, T., \& Bond, A. (2020). The difference between emergency remote teaching and online learning. Educause Review, 27, pp.1-12.

IESALC, U. (2020). COVID-19 y educación superior: de los efectos inmediatos al día después. http://www.iesalc.unesco.org/wp-content/uploads/2020/04/COVID-19-060420-ES2.pdf

INEGI (2019). Encuesta nacional sobre disponibilidad y uso de tecnologías de la información en los hogares (ENDUTIH). https://www.inegi.org.mx/temas/ticshogares/\#Tabulados

Marinoni, Giorgio, Van't Land, Hilligje, \& Jensen, Trine. (2020). The impact of COVID19 on higher education around the world IAU global survey report. https://www.iau-ziu.net/IMG/pdf/iau covid19 and he survey report final may 2020.pdf 
Mejía-Pérez, G., \& S. Worthman, S. (2018). La geografía de las oportunidades. El caso de las sedes de las universidades autónomas en municipios con poca oferta de educación superior. Revista Iberoamericana de Educación Superior, 8(23), pp.25-48. https://doi.org/10.22201/iisue.20072872e.2017.23.244

Ordorika, I. (2020). Pandemia y educación superior. Revista de la Educación Superior, 49(194), pp.1-8. http://resu.anuies.mx/ojs/index.php/resu/article/view/1120

Ramos Mancilla, O. (2019). Acceso a internet desigual y heterogéneo en los pueblos indígenas de la Sierra Norte de Puebla (México). Revista Española de Antropología Americana, 48, pp.9-27. https://doi.org/10.5209/REAA.63687

Rivera Polo, F., \& Rivera Vargas, P. (2018). Las universidades regionales ante el desafío de la descentralización. El caso de la Universidad de Aysén en Chile. Revista de La Educación Superior, 47(187), pp.49-70.

https://doi.org/10.36857/resu.2018.187.481

SEP (2020). Principales cifras del sistema educativo nacional 2019-2020. Autor:

México.https://www.planeacion.sep.gob.mx/Doc/estadistica_e_indicadores/principales_cifras/principales cifras 20192020 bolsillo.pdf

Silva, M. (2020). La dimensión pedagógica de la equidad en educación superior. Archivos Analíticos de Políticas Educativas, 28(46).

Toquero, C. M. (2021). Emergency remote education experiment amid COVID19 pandemic. IJERI: International Journal of Educational Research and Innovation, (15), pp.162-176.

World Bank (2020). Guidance note on remote learning and COVID-19 (English). World Bank Group.

http://documents.worldbank.org/curated/en/531681585957264427/Guidance-Note-onRemoteLearning-and-COVID-19

\section{BIONOTAS}

Abelardo Romero Fernández. Profesor-Investigador de Tiempo Completo en BUAP. Es licenciado en Lenguas Modernas (BUAP) y en Pedagogía (UNAM). Es Maestro en Educación Superior (ULSA). Actualmente realizada estudios de Doctorado en Educación (ULSA). Además, es PRODEP y parte del Cuerpo Académico BUAP-354.

Correo electrónico: abelardo.romero@correo.buap.mx https://orcid.org/0000-0002-1935-4365 
Laura Villanueva Méndez. Profesora-Investigadora de Tiempo Completo en BUAP. Es Licenciada en Psicología (BUAP) y Maestra en Psicología (UNAM).

Actualmente se encuentra realizando estudios de Doctorado en Educación (ULSA). Además, cuenta con la distinción PRODEP y es parte del Cuerpo Académico BUAP-354.

Correo electrónico: laura.villanueva@correo.buap.mx

https://orcid.org/0000-0002-1161-0379

Fabio Morandín Ahuerma. Profesor-Investigador de Tiempo Completo en BUAP.

Es Doctor en Filosofía (UV) y Maestro en Estudios Diplomáticos (Instituto Matías Romero). Cuenta con la distinción PRODEP y es parte del Cuerpo Académico BUAP-354.

Además, es autor de publicaciones científicas y de divulgación.

Correo electrónico: fabio.morandin@correo.buap.mx

https://orcid.org/0000-0001-6082-2207

Ana Liviere Vargas Vizuet. Profesora-Investigadora de Tiempo Completo en BUAP. Es Maestra en Historia (BUAP) y Licenciada en Etnología (ENAH). Ha publicado sobre el tema de Historia de la Salud y Medicina. Cuenta con la distinción PRODEP y es parte de Cuerpo Académico BUAP- 354.

Correo electrónico: analiviere.vargas@correo.buap.mx https://orcid.org/0000-0002-9506-8782 